\title{
Sizable excitonic effects undermining the photocatalytic efficiency of $\beta-\mathrm{Cu}_{2} \mathrm{~V}_{2} \mathrm{O}_{7}$
}

\author{
Julia Wiktor, ${ }^{*} \dagger$ Igor Reshetnyak, ${ }^{\dagger}$ Michal Strach, ${ }^{\ddagger}$ Mariateresa Scarongella, ${ }^{\ddagger}$ \\ Raffaella Buonsanti, ${ }^{\ddagger}$ and Alfredo Pasquarello ${ }^{\dagger}$
}

Chaire de Simulation à l'Echelle Atomique (CSEA), Ecole Polytechnique Fédérale de Lausanne (EPFL), CH-1015 Lausanne, Switzerland, and Laboratory of Nanochemistry for Energy (LNCE), Ecole Polytechnique Fédérale de Lausanne (EPFL), CH-1951 Sion, Switzerland

E-mail: julia.wiktor@epfl.ch

${ }^{*}$ To whom correspondence should be addressed

${ }^{\dagger}$ Chaire de Simulation à l'Echelle Atomique (CSEA), Ecole Polytechnique Fédérale de Lausanne (EPFL), CH-1015 Lausanne, Switzerland

${ }^{\ddagger}$ Laboratory of Nanochemistry for Energy (LNCE), Ecole Polytechnique Fédérale de Lausanne (EPFL), CH-1951 Sion, Switzerland 


\begin{abstract}
Copper vanadates have been proposed as promising photoanodes for water-splitting photoelectrochemical cells, but their performance has recently been shown to be severely limited. To understand this behavior, we study the electronic structure and the optical properties of $\beta-\mathrm{Cu}_{2} \mathrm{~V}_{2} \mathrm{O}_{7}$ both experimentally and computationally. The measured absorption spectrum shows an absorption peak at $1.5 \mathrm{eV}$ followed by the onset of an apparent continuum at $2.26 \mathrm{eV}$, as generally found for this class of materials. We perform calculations within the framework of the QSG $\tilde{W}$ method and the Bethe-Salpeter equation, while including effects of magnetic ordering, nuclear quantum motion, and thermal vibrations. We demonstrate the occurrence of two kinds of excitons with high binding energies upon optical excitation in $\beta-\mathrm{Cu}_{2} \mathrm{~V}_{2} \mathrm{O}_{7}$, which account for the first absorption peak and the lower edge of the apparent continuum. These results provide one possible explanation for the low photocatalytic efficiencies of copper vanadates, despite the favorable size of their optical band gaps.
\end{abstract}

\title{
Graphical TOC Entry
}

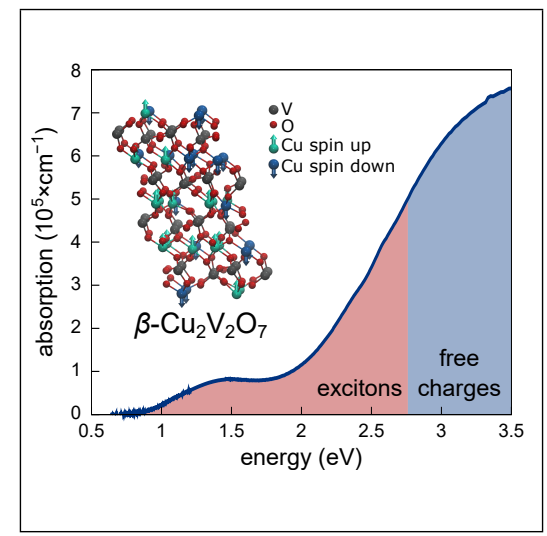


Photocatalytic water splitting is regarded as a promising way of generating renewable energy. ${ }^{1-3}$ To achieve high efficiencies of the reaction, it is essential to identify photoabsorbers with optimal properties. As a consequence, extensive efforts are being undertaken to identify suitable candidates. Recent high-throughput searches ${ }^{4,5}$ proposed copper vanadates as a promising class of materials for photocatalytic water splitting, mainly based on the favorable sizes of their band gaps. However, experimental studies have revealed that their performance is severely limited, ${ }^{6}$ even though their measured band gaps of about $2 \mathrm{eV}^{5-7}$ suggest that cells based on these materials should potentially reach high efficiencies. ${ }^{8}$ For the widely studied vanadate $\mathrm{BiVO}_{4}$, heterojunctions, ${ }^{9}$ cocatalyst deposition, ${ }^{10}$ gradient doping, ${ }^{11}$ and crystallographic orientation ${ }^{12}$ proved to be effective means to achieve photocurrent densities on par with the theoretical limit. Following the same rationale, a few experimental studies have been undertaken to optimize the morphology and doping of copper vanadates. ${ }^{13,14}$ However, such strategies have proven unsuccessful so far.

Though no definite conclusions have been drawn as to the origin of the low measured efficiencies in copper vanadates, it was suggested that high bulk recombination rates might be one of the underlying detrimental factors. ${ }^{14}$ Furthermore, a number of studies on copper vanadates have observed an unusual feature in the absorption spectrum, apparently unrelated to the band structure. This feature has remained poorly understood, possibly related to a localized ligand field excitation at the $\mathrm{Cu}^{2+}$ cation. ${ }^{15}$ While the corresponding states are generally not assumed to produce mobile charge carriers, ${ }^{15}$ this limited understanding nevertheless calls for a more thorough investigation of the optical properties of copper vanadates.

In the present study, we investigate, both computationally and experimentally, the electronic structure and the optical properties of $\beta-\mathrm{Cu}_{2} \mathrm{~V}_{2} \mathrm{O}_{7}$ (ziesite phase), as a representative of the copper vanadate class. We demonstrate that strong excitonic effects account for both the first peak in the absorption spectrum and the onset of the apparent continuum. These results explain the limited photocatalytic efficiency of photoelectrochemical cells based on 
copper vanadates.

a)

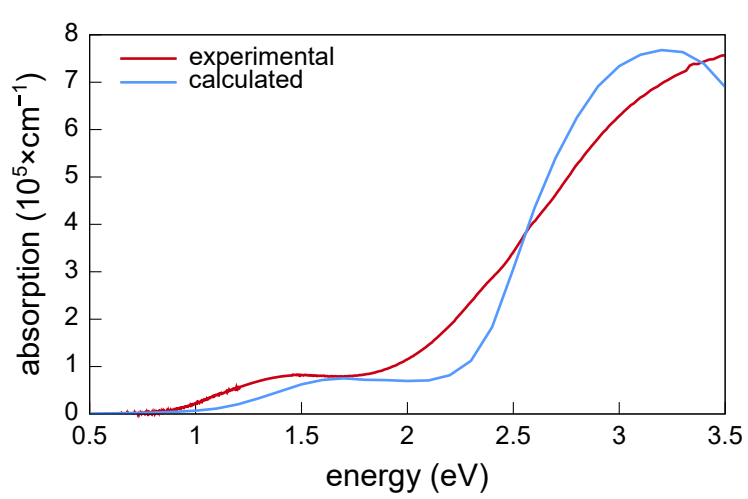

b)

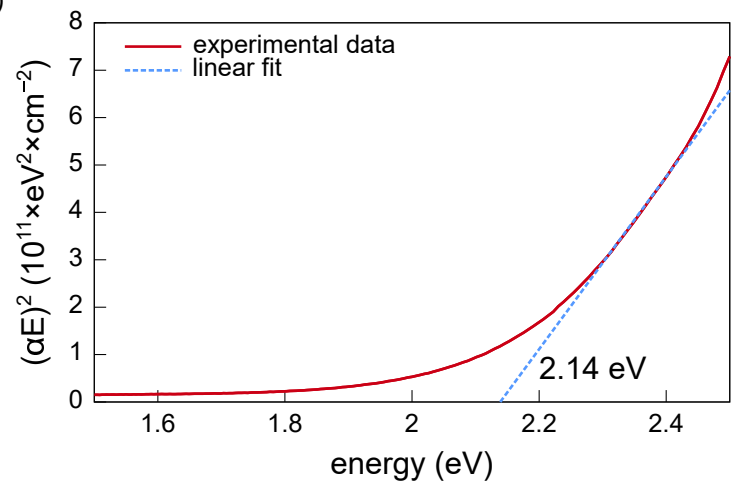

Figure 1: a) Experimental absorption coefficient compared to that achieved through our computational scheme. b) Experimental Tauc plot.

We first determine experimentally the optical absorption spectrum of $\beta-\mathrm{Cu}_{2} \mathrm{~V}_{2} \mathrm{O}_{7}$ using UV-vis spectroscopy. Thin films of $\mathrm{Cu}_{2} \mathrm{~V}_{2} \mathrm{O}_{7}$ were prepared using the seeded growth approach. The sample composition was verified by X-ray diffraction measurements, as detailed in the Supporting Information (SI). The corresponding absorption spectrum is given in Fig. 1 a). The raw data contained severe noise around $1.4 \mathrm{eV}$ due to a change of lamps in our dual light source spectrometer during the measurement (cf. SI), and the curve was treated with a locally weighted scatterplot smoothing (LOESS) method. Additionally, the background was removed to ease comparison. The measured absorption spectrum shows a first peak at about $1.5 \mathrm{eV}$ and the onset of an apparent continuum close to $2 \mathrm{eV}$. These features are generally observed in the class of copper oxides with $\mathrm{Cu}^{2+}$ ions, ${ }^{5-7,15,16}$ corroborating their intrinsic origin. From the corresponding Tauc plot [Fig. 1 b)], we estimate a value of $2.14 \mathrm{eV}$ for this onset.

While steady-state spectra are routinely used to evaluate the absorption properties of semiconductors, they do not provide any information about the nature of the generated charge carriers. To evaluate the electronic properties underlying the observed features, we resort to a first-principles modeling scheme. From the computational point of view, modeling a complex material such as a copper vanadate requires special care. First, the occurrence 
of the $2+$ oxidation state of $\mathrm{Cu}$ leads to the presence of unpaired electrons and to magnetic ordering. In particular, $\beta-\mathrm{Cu}_{2} \mathrm{~V}_{2} \mathrm{O}_{7}$ is known to be paramagnetic at room temperature, ${ }^{17,18}$ which presents a challenge for first-principles modeling. ${ }^{19,20}$ Moreover, it has recently been observed that the electronic structure of an analogous vanadate, $\mathrm{BiVO}_{4}$, is strongly affected by thermal vibrations and nuclear quantum motions. ${ }^{21}$ These effects might similarly influence the electronic structure of $\beta-\mathrm{Cu}_{2} \mathrm{~V}_{2} \mathrm{O}_{7}$, and thus need to be properly accounted for in the calculations. Finally, the full treatment of the optical properties of the material requires the consideration of excitonic effects, which generally involve advanced schemes such as the Bethe-Salpeter equation.

We begin the theoretical analysis by studying the electronic structure of $\beta-\mathrm{Cu}_{2} \mathrm{~V}_{2} \mathrm{O}_{7}$ in the antiferromagnetic ground state at $0 \mathrm{~K} \cdot{ }^{22}$ We perform calculations within many-body perturbation theory through the use of the self-consistent QSGW technique. An efficient exchange-correlation kernel is included to account for vertex corrections, as described in Ref. 23. This approach has been shown to yield band gaps in very good agreement with experiment. ${ }^{23,24}$ We refer the reader to the SI for computational details. In Fig. 2, we show the density of states (DOS) as calculated with various electronic structure schemes. The top of the valence band results from a mixture of $\mathrm{O} 2 p, \mathrm{~V} 3 d$, and $\mathrm{Cu} 3 d$ orbitals, while the lowest conduction band states essentially consist of $\mathrm{Cu} 3 d$ states and give rise to an isolated peak in the DOS. This is consistent with the $d^{9}$ occupation of the $\mathrm{Cu} 3 d$ states in the $\mathrm{Cu}^{2+}$ ion. The QSGE calculation gives an indirect band gap of $3.56 \mathrm{eV}$, whereas the direct band gap is found to lie slightly higher at $3.63 \mathrm{eV}$. The semilocal Perdew-Burke-Ernzerhof (PBE) functional yields a much lower band gap of $0.72 \mathrm{eV}$, but also a much larger separation between the isolated peak of empty $\mathrm{Cu} 3 d$ states and the higher lying conduction band states. We note that the $\mathrm{QS} G \tilde{W}$ band gap at $0 \mathrm{~K}(3.56 \mathrm{eV})$ is much higher than the onset at 2.14 $\mathrm{eV}$ of the apparent continuum in the experimental spectrum (Fig. 1). However, following recent work on $\mathrm{BiVO}_{4},{ }^{21}$ one expects additional effects, such as nuclear quantum motions, thermal vibrations, and excitonic effects, to lower the theoretical estimate. To address larger 
supercells preserving the accuracy of the $\mathrm{QS} G \tilde{W}$ scheme, it is convenient to make use of a hybrid functional. For this purpose, we use the Perdew-Burke-Ernzerhof (PBE0) hybrid functional ${ }^{25}$ with a mixing parameter $\alpha$ set to 0.225 in order to closely reproduce the DOS calculated in $\mathrm{QS} G \tilde{W}$ (see Fig. 2).

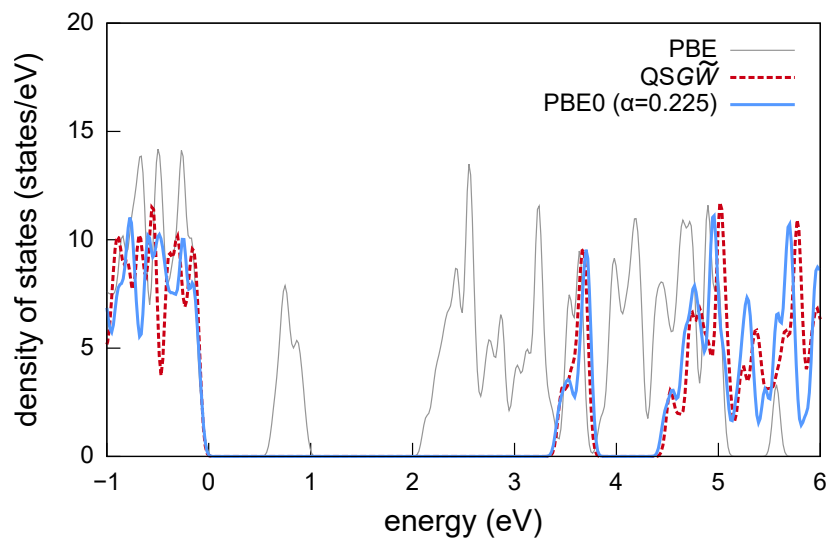

Figure 2: Density of states for the antiferromagnetic ground state of $\beta-\mathrm{Cu}_{2} \mathrm{~V}_{2} \mathrm{O}_{7}$ at $0 \mathrm{~K}$, as calculated using the $\operatorname{PBE}, \operatorname{QS} G \tilde{W}$, and $\operatorname{PBE} 0(\alpha=0.225)$ methods. The energies are referred to the top of the valence band for each calculation. We use a broadening of $0.05 \mathrm{eV}$ to generate the plots.

$\beta-\mathrm{Cu}_{2} \mathrm{~V}_{2} \mathrm{O}_{7}$ has been shown to be paramagnetic at room temperature. ${ }^{17,18}$ To describe the paramagnetic state within density functional theory, we use the Disordered Local Moment model,${ }^{26,27}$ in which a random distribution of atomic magnetic moments is assumed. Here, we consider only collinear magnetic moments, which is appropriate in this case as spin-orbit coupling is not dominant. ${ }^{28}$ To achieve a random distribution of spin-up and spin-down $\mathrm{Cu}$ atoms in $\beta-\mathrm{Cu}_{2} \mathrm{~V}_{2} \mathrm{O}_{7}$, we generate a special quasirandom structure (SQS) ${ }^{29}$ containing 176 atoms in the supercell, as represented in Fig. 3 a). This cell contains $32 \mathrm{Cu}$ atoms, 16 of them with spin up and 16 with spin down. When we compare the density of states of the antiferromagnetic and paramagnetic states calculated with the PBE functional at a temperature of $0 \mathrm{~K}$, we find that the magnetic structure of the paramagnetic state leads to a broadening of the width of the empty $\mathrm{Cu} 3 d$ states, consequently reducing the band gap by $0.18 \mathrm{eV}$.

Next, we study the influence of thermal disorder and nuclear quantum effects (NQEs) on 
the band gap of $\beta-\mathrm{Cu}_{2} \mathrm{~V}_{2} \mathrm{O}_{7}$. We carry out path-integral molecular dynamics (PIMD) at the PBE level for 5 ps at $300 \mathrm{~K}$, within the NVT ensemble. The simulation is performed for the paramagnetic supercell containing 176 atoms. To achieve an accurate description of the electronic structure, we perform subsequent calculations with the hybrid functional PBE0 $(\alpha=0.225)^{25}$ on 300 configurations regularly spaced in time from the PIMD trajectory. The density of states calculated for the selected paramagnetic configurations is shown in Fig. 3 b), where it is compared to the corresponding DOS of the antiferromagnetic ground state at $0 \mathrm{~K}$, achieved with the same hybrid functional but neglecting NQEs. From the DOS, we extract a room-temperature fundamental band gap of $2.76 \mathrm{eV}$, smaller than the value at $0 \mathrm{~K}(3.56 \mathrm{eV})$ by almost $0.8 \mathrm{eV}$. We note that in the DOS of the paramagnetic structure at $300 \mathrm{~K}$ the empty $\mathrm{Cu} 3 d$ states merge with the higher lying conduction states. This qualitative aspect is missed in the semilocal description at $0 \mathrm{~K}$, undermining the reliability of such a scheme in high-throughput searches of new materials.
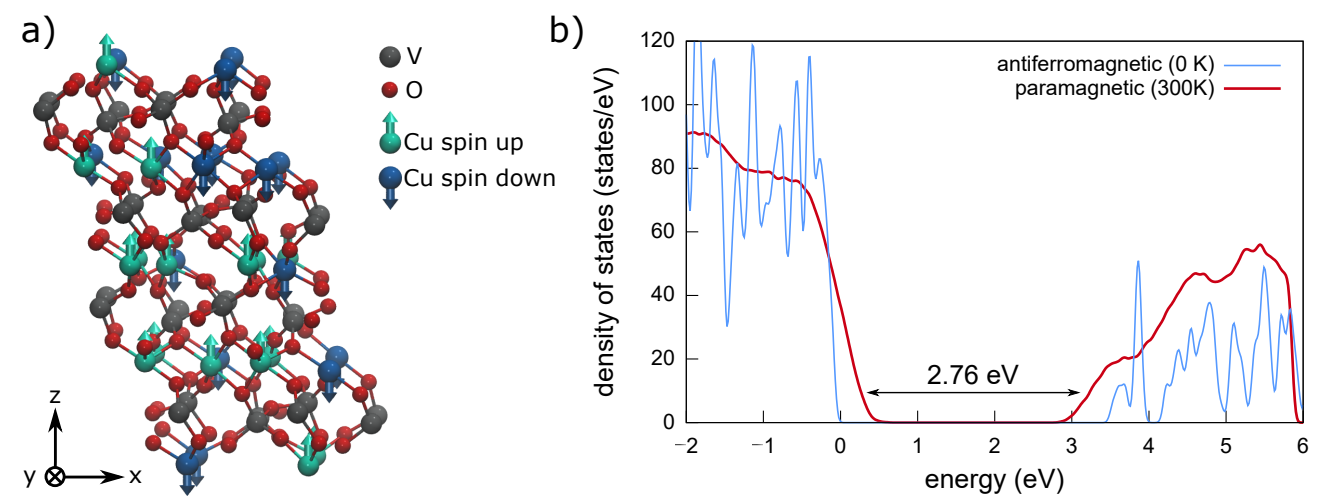

Figure 3: a) Paramagnetic supercell of $\beta-\mathrm{Cu}_{2} \mathrm{~V}_{2} \mathrm{O}_{7}$ containing a quasirandom distribution of spin-up (green) and spin-down (blue) $\mathrm{Cu}$ atoms, based on a special quasirandom structure (SQS). b) Density of states of $\beta-\mathrm{Cu}_{2} \mathrm{~V}_{2} \mathrm{O}_{7}$ at $300 \mathrm{~K}$, in the paramagnetic state. The calculation uses the $\operatorname{PBE} 0(\alpha=0.225)$ hybrid functional and includes NQEs. For comparison, we also show the DOS of the antiferromagnetic ground state, calculated with the same functional at $0 \mathrm{~K}$ but neglecting NQEs. The DOS are aligned using the $\mathrm{Cu} 3 s$ states. The energies are referred to the top of the valence band of the antiferromagnetic state. We use a broadening of $0.05 \mathrm{eV}$ to generate the plots.

Optical absorption spectra can be used to extract the optical band gap of $\beta-\mathrm{Cu}_{2} \mathrm{~V}_{2} \mathrm{O}_{7}$. This property differs from the fundamental band gap by the electron-hole interaction. 
To account for excitonic effects, we solve the Bethe-Salpeter equation (BSE) ${ }^{30}$ using the ABINIT $^{31-33}$ and DP-CODE ${ }^{34}$ packages. We calculate the absorption spectra on 20 instantaneous configurations from the PIMD simulation. In this way, our description accounts for the paramagnetic spin structure, the thermal vibrations, and the nuclear quantum motions. Since the treatment of the 176-atom cell is demanding, we use wave functions achieved at the PBE level and a scissor operator of $2.8 \mathrm{eV}$, which is chosen to reproduce the $\mathrm{QS} G \tilde{W}$ band gap of the antiferromagnetic ground state at $0 \mathrm{~K}$. In the SI, the validity of this approach is analyzed and the computational details are provided.
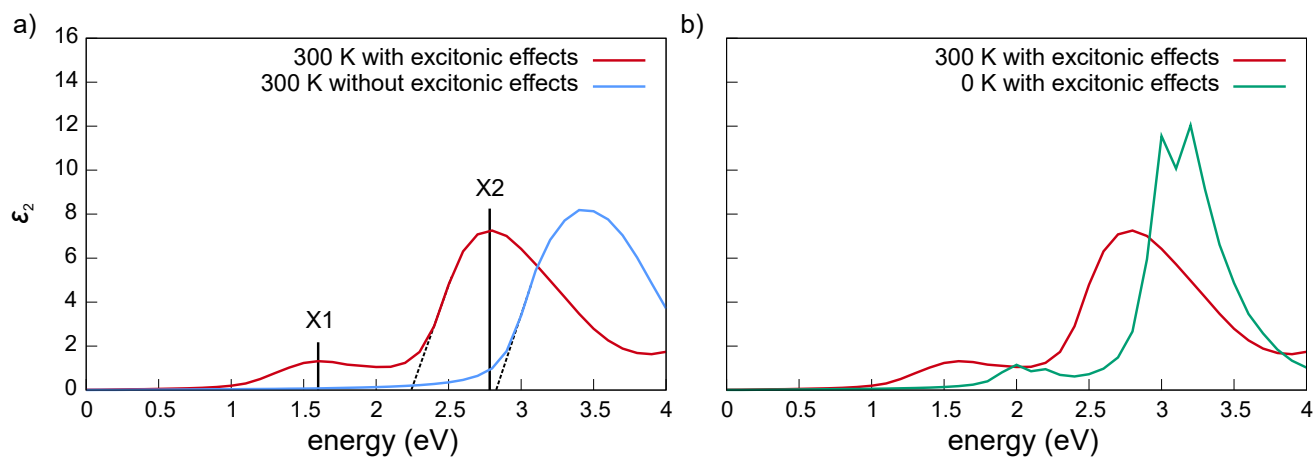

Figure 4: a) Comparison between the imaginary part of the dielectric function $\left(\varepsilon_{2}\right)$ calculated with the BSE for the paramagnetic ground state at $300 \mathrm{~K}$ (blue) and the corresponding result obtained in the RPA (blue). The results include NQEs. b) Comparison between the imaginary part of the dielectric function calculated with the BSE for the paramagnetic ground state at $300 \mathrm{~K}$ (including NQEs) and the result achieved at $0 \mathrm{~K}$ for the antiferromagnetic state (green).

We compare in Fig. 4 a) the imaginary part of the dielectric function $\varepsilon_{2}$ calculated by solving the BSE equation with that obtained within the random phase approximation (RPA), which does not include excitonic effects. The RPA curve shows a peak composed of excitations to $\mathrm{Cu} 3 d$ states at $3.5 \mathrm{eV}$ with an onset at $2.76 \mathrm{eV}$, consistent with the fundamental band gap in Fig. 3. The comparison with the BSE result shows that the excitonic effects are sizable in $\beta-\mathrm{Cu}_{2} \mathrm{~V}_{2} \mathrm{O}_{7}$. We distinguish two peaks below the fundamental band gap at 1.6 and $2.7 \mathrm{eV}$, denoted as X1 and X2, respectively. Since these excitonic features correspond to excitations to $\mathrm{Cu} 3 d$ states, we refer their peak positions with respect to the RPA peak and 
find respective binding energies of 1.9 and $0.8 \mathrm{eV}$. We determine the onset of the feature $\mathrm{X} 2$ to be at $2.26 \mathrm{eV}$. This shows a sizable shift of $0.5 \mathrm{eV}$ with respect to the RPA one, in accord with shifts of similar size observed in other complex copper oxides, such as $\mathrm{CuAlO}_{2} \cdot{ }^{35,36}$ This can be considered as the minimal energy that needs to be supplied to overcome the electronhole binding energy and to achieve mobile carriers. We remark that there is a nontrivial interplay between atomic displacements and excitonic effects. This can be inferred from Fig. $4 \mathrm{~b}$ ), where $\varepsilon_{2}$ calculated for the antiferromagnetic ground state at $0 \mathrm{~K}$ and for the paramagnetic state at $300 \mathrm{~K}$ are compared. A simplistic approach consisting in applying the broadening due to atomic displacements observed in Fig. 3 to the result at $0 \mathrm{~K}$ could not give the correct one at $300 \mathrm{~K}$.

a)

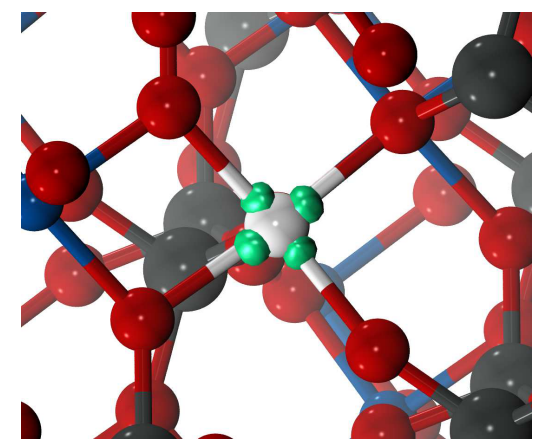

b)

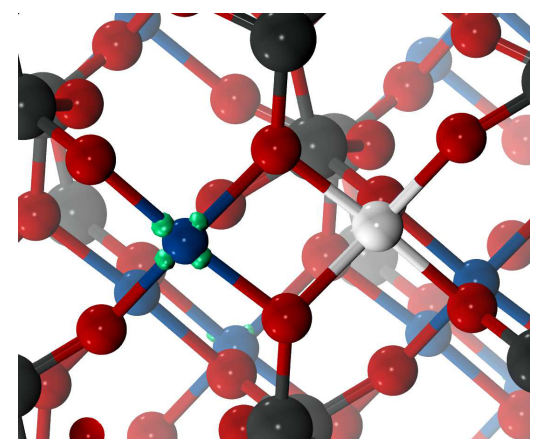

Figure 5: Representation of the wave functions of the lowest lying excitonic features (a) X1 and (b) $\mathrm{X} 2$ in $\beta-\mathrm{Cu}_{2} \mathrm{~V}_{2} \mathrm{O}_{7} . \mathrm{Cu}, \mathrm{O}$, and $\mathrm{V}$ atoms are in blue, red, and gray, respectively. To illustrate the two-particle wave functions of the excitons, the spatial coordinate of the hole has been fixed at the $\mathrm{Cu}$ atom marked in white, while the orbital of the electron wave function is displayed by a green contour.

To analyze the nature of these two excitonic states, we plot the corresponding wave functions in Fig. 5. We observe that the first peak X1 is dominated by on-site $\mathrm{Cu}-\mathrm{Cu}$ transitions between occupied and empty $3 d$ states. These transitions are forbidden in a spherically symmetric environment, but acquire oscillation strength due to the crystal field, which is already present at $0 \mathrm{~K}$ in $\beta-\mathrm{Cu}_{2} \mathrm{~V}_{2} \mathrm{O}_{7}$ and is further enhanced at $300 \mathrm{~K}$. The excitonic feature X2, at higher energies, corresponds to excitations in which the electron and the hole reside in $\mathrm{Cu} 3 d$ orbitals of neighboring atoms. Hence, the two excitonic features bear from 
transitions to empty $\mathrm{Cu} 3 d$, as one could infer from the low-lying conduction band states in the DOS (Fig. 3). The broadening due to the atomic motion is then responsible for merging the X2 feature with transitions to higher lying conduction band states.

To make a comparison with experiment, we construct the frequency-dependent absorption coefficient $\alpha(\omega)$

$$
\alpha(\omega)=\frac{4 \pi \omega}{c} \sqrt{\frac{\sqrt{\varepsilon_{1}^{2}(\omega)+\varepsilon_{2}^{2}(\omega)}-\varepsilon_{1}(\omega)}{2}},
$$

where $c$ is the speed of light, $\varepsilon_{2}$ the imaginary part of the calculated dielectric function of the paramagnetic state at $300 \mathrm{~K}$ (given in Fig. 4) and $\varepsilon_{1}$ the corresponding real part (given in the SI). The calculated $\alpha(\omega)$ is compared to our measured absorption spectrum in Fig. 1 a). The agreement between theory and experiment is remarkable, the peak position of X1 and the onset of $\mathrm{X} 2$ being both well reproduced. This confirms the validity of the underlying theoretical scheme and highlights the importance of all steps taken to achieve the present description.

The good agreement between theory and experiment also allows us to draw important conclusions as to the interpretation of the features in the absorption spectrum of $\beta-\mathrm{Cu}_{2} \mathrm{~V}_{2} \mathrm{O}_{7}$ and to the general suitability of this class of materials in photocatalytic devices. Our results indicate that the peculiar feature associated with the lowest-energy transitions originates from on-site transitions at the $\mathrm{Cu}^{2+}$ sites, corresponding to excitons of high binding energy. However, since the weak absorption in correspondence to this feature has not been considered in efficiency assessments, its origin is inconsequential to the estimation of the performance of this semiconductor. As for the higher lying excitations, the present results indicate that the apparent onset of the continuum actually corresponds to transitions to bound excitons in which the photogenerated charges are localized on neighboring $\mathrm{Cu}$ sites. Therefore, these charges are not available for catalytic reactions at the surface, unless an additional energy of at least $0.5 \mathrm{eV}$ is supplied to split the charges. This value is much higher than found for other metal oxides, such as $\mathrm{BiVO}_{4}$, for which an excitonic binding energy of only $0.11 \mathrm{eV}$ 
has been calculated, ${ }^{21}$ and undermines the viability of using $\beta-\mathrm{Cu}_{2} \mathrm{~V}_{2} \mathrm{O}_{7}$ in a PEC cell. The significant excitonic binding energy might contribute to the high onset potentials measured for pristine $\mathrm{Cu}-\mathrm{V}-\mathrm{O}$ samples. ${ }^{7,13}$

To conclude, we obtained an exceptionally good agreement between the measured and modeled optical absorption spectra of $\beta-\mathrm{Cu}_{2} \mathrm{~V}_{2} \mathrm{O}_{7}$, a representative of the class of copper vanadate compounds, presently widely investigated in view of photocatalytical applications. Our theoretical modeling relied on state-of-the-art computational methods, including pathintegral molecular dynamics, self-consistent many-body perturbation theory, and the BetheSalpeter equation. Our results highlight the occurrence of excitons of high binding energy in the absorption spectrum as one of the reasons undermining the performance of $\beta-\mathrm{Cu}_{2} \mathrm{~V}_{2} \mathrm{O}_{7}$ as a photoanode in a water splitting cell. As this limitation directly stems from the properties of the $\mathrm{Cu}^{2+}$ ions, these conclusions generalize to the entire class of copper vanadates. Hence, our work suggests that electron-hole interactions between photogenerated carriers should be systematically accounted for in future searches for new materials for water splitting.

\section{Acknowledgement}

The authors acknowledge financial support from the Swiss National Science Foundation (SNSF) (Grant No. 200020-172524). This work has been realized in relation to the National Center of Competence in Research (NCCR) "Materials' Revolution: Computational Design and Discovery of Novel Materials (MARVEL)" of the SNSF. We used computational resources of CSCS and SCITAS-EPFL.

\section{Supporting Information Available}

Experimental details on sample preparation, X-ray diffraction and UV-vis spectroscopy mea-

surements, computational details on QSG $\tilde{W}$ and BSE calculations, and additional Ref. 37-46 


\section{References}

(1) Fujishima, A.; Honda, K. Electrochemical Photolysis of Water at a Semiconductor Electrode. Nature 1972, 238, 37-38.

(2) Maeda, K.; Teramura, K.; Lu, D.; Takata, T.; Saito, N.; Inoue, Y.; Domen, K. Photocatalyst Releasing Hydrogen from Water. Nature 2006, 440, 295-295.

(3) Walter, M. G.; Warren, E. L.; McKone, J. R.; Boettcher, S. W.; Mi, Q.; Santori, E. A.; Lewis, N. S. Solar Water Splitting Cells. Chem. Rev. 2010, 110, 6446.

(4) Zhou, L.; Yan, Q.; Shinde, A.; Guevarra, D.; Newhouse, P. F.; Becerra-Stasiewicz, N.; Chatman, S. M.; Haber, J. A.; Neaton, J. B.; Gregoire, J. M. High Throughput Discovery of Solar Fuels Photoanodes in the $\mathrm{CuO}-\mathrm{V}_{2} \mathrm{O}_{5}$ System. Adv. Energy Mater. 2015, 5.

(5) Yan, Q.; Yu, J.; Suram, S. K.; Zhou, L.; Shinde, A.; Newhouse, P. F.; Chen, W.; Li, G.; Persson, K. A.; Gregoire, J. M. et al. Solar Fuels Photoanode Materials Discovery by Integrating High-Throughput Theory and Experiment. Proc. Natl. Acad. Sci. 2017, $114,3040-3043$.

(6) Jiang, C.-M.; Segev, G.; Hess, L. H.; Liu, G.; Zaborski, G.; Toma, F. M.; Cooper, J. K.; Sharp, I. D. Composition-Dependent Functionality of Copper Vanadate Photoanodes. ACS Appl. Mater. Interfaces 2018, 10627-10633.

(7) Gadiyar, C.; Strach, M.; Schouwink, P.; Loiudice, A.; Buonsanti, R. Chemical Transformations at the Nanoscale: Nanocrystal-Seeded Synthesis of $\beta-\mathrm{Cu}_{2} \mathrm{~V}_{2} \mathrm{O}_{7}$ with Enhanced Photoconversion Efficiencies. Chem. Sci. 2018, 5658-5665.

(8) Werner, J. H.; Kolodinski, S.; Queisser, H. J. Novel Optimization Principles and Efficiency Limits for Semiconductor Solar Cells. Phys. Rev. Lett. 1994, 72, 3851. 
(9) Zhou, Y.; Zhang, L.; Lin, L.; Wygant, B. R.; Liu, Y.; Zhu, Y.; Zheng, Y.; Mullins, C. B.; Zhao, Y.; Zhang, X. et al. Highly Efficient Photoelectrochemical Water Splitting from Hierarchical $\mathrm{WO}_{3} / \mathrm{BiVO}_{4}$ Nanoporous Sphere Arrays. Nano Letters 2017, 17, 80128017, PMID: 29185764.

(10) Abdi, F. F.; Firet, N.; van de Krol, R. Efficient BiVO 4 Thin Film Photoanodes Modified with Cobalt Phosphate Catalyst and W-doping. Chem. Cat. Chem. 5, 490-496.

(11) Abdi, F.; Han, L.; Smets, A.; Zeman, M.; Dam, B.; van de Krol, R. Efficient Solar Water Splitting by Enhanced Charge Separation in a Bismuth Vanadate-Silicon Tandem Photoelectrode. Nat. Commun. 2013, 4, 1-7.

(12) Han, H. S.; Shin, S.; Kim, D. H.; Park, I. J.; Kim, J. S.; Huang, P.-S.; Lee, J.-K.; Cho, I. S.; Zheng, X. Boosting the Solar Water Oxidation Performance of a $\mathrm{BiVO}_{4}$ Photoanode by Crystallographic Orientation Control. Energy Environ. Sci. 2018, 11, $1299-1306$.

(13) Guo, W.; Chemelewski, W. D.; Mabayoje, O.; Xiao, P.; Zhang, Y.; Mullins, C. B. Synthesis and Characterization of $\mathrm{CuV}_{2} \mathrm{O}_{6}$ and $\mathrm{Cu}_{2} \mathrm{~V}_{2} \mathrm{O}_{7}$ : Two Photoanode Candidates for Photoelectrochemical Water Oxidation. J. Phys. Chem. C 2015, 119, 27220-27227.

(14) Lumley, M. A.; Choi, K.-S. Investigation of Pristine and (Mo, W)-Doped $\mathrm{Cu}_{1} 1 \mathrm{~V}_{6} \mathrm{O}_{2} 6$ for Use as Photoanodes for Solar Water Splitting. Chem. Mater. 2017, 29, 9472-9479.

(15) Jiang, C.-M.; Farmand, M.; Wu, C. H.; Liu, Y.-S.; Guo, J.; Drisdell, W. S.; Cooper, J. K.; Sharp, I. D. Electronic Structure, Optoelectronic Properties, and Photoelectrochemical Characteristics of $\gamma-\mathrm{Cu}_{3} \mathrm{~V}_{2} \mathrm{O}_{8}$ Thin Films. Chem. Mater. 2017, 29, $3334-3345$.

(16) Lhermitte, C. R.; Bartlett, B. M. Advancing the Chemistry of $\mathrm{CuWO}_{4}$ for Photoelectrochemical Water Oxidation. Acc. Chem. Res. 2016, 49, 1121-1129. 
(17) Ponomarenko, L.; Vasil'ev, A.; Antipov, E.; Velikodny, Y. A. Magnetic Properties of $\mathrm{Cu}_{2} \mathrm{~V}_{2} \mathrm{O}_{7}$. Physica B 2000, 284, 1459-1460.

(18) He, Z.; Ueda, Y. Paramagnetic Anisotropy and Spin-Flop Transition in Single Crystals of the Quasi-One-Dimensional System $\beta-\mathrm{Cu}_{2} \mathrm{~V}_{2} \mathrm{O}_{7}$. Phys. Rev. B 2008, 77, 052402.

(19) Dorado, B.; Garcia, P. First-principles DFT + U modeling of actinide-based alloys: Application to paramagnetic phases of $\mathrm{UO}_{2}$ and $(\mathrm{U}, \mathrm{Pu})$ mixed oxides. Phys. Rev. $B$ 2013, 87, 195139.

(20) Abrikosov, I. A.; Ponomareva, A.; Steneteg, P.; Barannikova, S.; Alling, B. Recent Progress in Simulations of the Paramagnetic State of Magnetic Materials. Curr. Opin. Solid State Mater. Sci. 2016, 20, 85-106.

(21) Wiktor, J.; Reshetnyak, I.; Ambrosio, F.; Pasquarello, A. Comprehensive Modeling of the Band Gap and Absorption Spectrum of $\mathrm{BiVO}_{4}$. Phys. Rev. Mater. 2017, 1, 022401.

(22) Yashima, M.; Suzuki, R. O. Electronic Structure and Magnetic Properties of Monoclinic $\beta-\mathrm{Cu}_{2} \mathrm{~V}_{2} \mathrm{O}_{7}$ : A GGA+U Study. Phys. Rev. B 2009, 79, 125201.

(23) Chen, W.; Pasquarello, A. Accurate Band Gaps of Extended Systems Via Efficient Vertex Corrections in GW. Phys. Rev. B 2015, 92, 041115.

(24) Wiktor, J.; Rothlisberger, U.; Pasquarello, A. Predictive Determination of Band Gaps of Inorganic Halide Perovskites. J. Phys. Chem. Lett. 2017, 8, 5507-5512.

(25) Perdew, J. P.; Ernzerhof, M.; Burke, K. Rationale for Mixing Exact Exchange with Density Functional Approximations. J. Chem. Phys. 1996, 105, 9982-9985.

(26) Hubbard, J. The Magnetism of Iron. Phys. Rev. B 1979, 19, 2626.

(27) Hasegawa, H. Single-site Spin Fluctuation Theory of Itinerant-Electron Systems with Narrow Bands. II. Iron and nickel. J. Phys. Soc. Jpn 1980, 49, 963-971. 
(28) Gyorffy, B.; Pindor, A.; Staunton, J.; Stocks, G.; Winter, H. A First-Principles Theory of Ferromagnetic Phase Transitions in Metals. J. Phys. F: Met. Phys. 1985, 15, 1337.

(29) Zunger, A.; Wei, S.-H.; Ferreira, L.; Bernard, J. E. Special Quasirandom Structures. Phys. Rev. Lett. 1990, 65, 353.

(30) Cutkosky, R. E. Solutions of a Bethe-Salpeter Equation. Phys. Rev. 1954, 96, 11351141.

(31) Gonze, X.; Beuken, J.-M.; Caracas, R.; Detraux, F.; Fuchs, M.; Rignanese, G.-M.; Sindic, L.; Verstraete, M.; Zerah, G.; Jollet, F. et al. First-Principles Computation of Material Properties: The ABINIT Software Project. Comput. Mater. Sci. 2002, 25, $478-492$.

(32) Gonze, X.; Amadon, B.; Anglade, P.-M.; Beuken, J.-M.; Bottin, F.; Boulanger, P.; Bruneval, F.; Caliste, D.; Caracas, R.; Côté, M. et al. ABINIT: First-Principles Approach to Material and Nanosystem Properties. Comput. Phys. Commun. 2009, 180, $2582-2615$.

(33) Gonze, X.; Jollet, F.; Araujo, F. A.; Adams, D.; Amadon, B.; Applencourt, T.; Audouze, C.; Beuken, J.-M.; Bieder, J.; Bokhanchuk, A. et al. Recent Developments in the ABINIT Software Package. Comput. Phys. Commun. 2016, 205, 106 - 131.

(34) Olevano, V.; Reining, L.; Sottile, F. DP-Code, Http://www.dp-Code.org/. http:// www.dp-code. org/.

(35) Laskowski, R.; Christensen, N. E.; Blaha, P.; Palanivel, B. Strong Excitonic Effects in $\mathrm{CuAlO}_{2}$ Delafossite Transparent Conductive Oxides. Phys. Rev. B 2009, 79, 165209.

(36) Vidal, J.; Trani, F.; Bruneval, F.; Marques, M. A.; Botti, S. Effects of Electronic and Lattice Polarization on the Band Structure of Delafossite Transparent Conductive Oxides. Phys. Rev. Lett. 2010, 104, 136401. 
(37) Hamann, D. Optimized Norm-Conserving Vanderbilt Pseudopotentials. Phys. Rev. B 2013, 88, 085117.

(38) Hugues, J. M.; Brown, M. A. The Crystal Structure of Ziesite, $\beta-\mathrm{Cu}_{2} \mathrm{~V}_{2} \mathrm{O}_{7}$, a Thortveitite-Type Structure with a Non-Linear X-O-X Inter-Tetrahedral Bond. Neu. Jb. Mineral. Mh. 1989, 41-47.

(39) Perdew, J. P.; Burke, K.; Ernzerhof, M. Generalized Gradient Approximation Made Simple. Phys. Rev. Lett. 1996, 77, 3865.

(40) Lebègue, S.; Arnaud, B.; Alouani, M.; Bloechl, P. Implementation of an All-Electron GW Approximation Based on the Projector Augmented Wave Method Without Plasmon Pole Approximation: Application to $\mathrm{Si}, \mathrm{SiC}, \mathrm{AlAs}$, InAs, NaH, and KH. Phys. Rev. B 2003, 67, 155208.

(41) VandeVondele, J.; Krack, M.; Mohamed, F.; Parrinello, M.; Chassaing, T.; Hutter, J. Quickstep: Fast and Accurate Density Functional Calculations Using a Mixed Gaussian and Plane Waves Approach. Comput. Phys. Commun. 2005, 167, 103 - 128.

(42) Ceriotti, M.; Bussi, G.; Parrinello, M. Nuclear Quantum Effects in Solids Using a Colored-Noise Thermostat. Phys. Rev. Lett. 2009, 103, 030603.

(43) VandeVondele, J.; Hutter, J. Gaussian Basis Sets for Accurate Calculations on Molecular Systems in Gas and Condensed Phases. J. Chem. Phys. 2007, 127, 114105.

(44) Goedecker, S.; Teter, M.; Hutter, J. Separable Dual-Space Gaussian Pseudopotentials. Phys. Rev. B 1996, 54, 1703-1710.

(45) Fuchs, F.; Rödl, C.; Schleife, A.; Bechstedt, F. Efficient O(N2) Approach to Solve the Bethe-Salpeter Equation for Excitonic Bound States. Phys. Rev. B 2008, 78, 085103.

(46) Hung, L.; Bruneval, F.; Baishya, K.; Ögüt, S. Benchmarking the GW Approximation 
and Bethe-Salpeter Equation for Groups IB and IIB Atoms and Monoxides. J. Chem. Theory Comput. 2017, 13, 2135-2146. 